\title{
Improving the Identification of Out-of-Hospital Sudden Cardiac Deaths in a General Practice Research Database
}

\author{
Cristina Varas-Lorenzo ${ }^{1} \cdot$ Alejandro Arana $^{1}$ (D) Catherine B. Johannes ${ }^{2} \cdot$ \\ Lisa J. McQuay ${ }^{3} \cdot$ Kenneth J. Rothman $^{2}$ - Daniel Fife ${ }^{4}$
}

Published online: 2 August 2016

(c) The Author(s) 2016. This article is published with open access at Springerlink.com

\begin{abstract}
Background The ascertainment of sudden cardiac death (SCD) in electronic health databases is challenging.

Objectives Our objective was to evaluate the applicability of the validated computer definition of SCD developed by Chung et al. in a retrospective study of SCD and domperidone exposure in the Clinical Practice Research Datalink (CPRD). Methods We assessed out-of-hospital SCD by applying the validated computer definition and linking data with Hospital Episode Statistics and death certificates. We developed a separate algorithm to identify end-of-life care in noninstitutionalized patients and excluded associated deaths from the analysis to address their misclassification as SCD.

Results Of the 681,104 patients in the study cohort, 3444 were initially classified as out-of-hospital SCD. Next, 163 deaths were identified as expected deaths by our algorithm for end-oflife home care. After review of patient profiles, 162 were classified as expected deaths because of evidence that the patient received palliative or end-of-life care, but one was a false
\end{abstract}

Electronic supplementary material The online version of this article (doi:10.1007/s40801-016-0086-1) contains supplementary material, which is available to authorized users.

Alejandro Arana

aarana@rti.org

1 Epidemiology, RTI Health Solutions, Trav. Gracia 56 Atico 1, 08006 Barcelona, Spain

2 Epidemiology, RTI Health Solutions, 1440 Main Street, Suite 310, Waltham, MA 02451, USA

3 Epidemiology, RTI Health Solutions, 200 Park Offices Drive, Research Triangle Park, NC 27709, USA

4 Pharmaceutical Research and Development, Janssen Research \& Development, LLC, 1125 Trenton-Harbourton Road, Titusville, NJ 08560, USA negative. The exclusion of such cases appreciably changed the odds ratio for current exposure to domperidone compared with non-use of study medications from 2.09 (95\% confidence interval [CI] 1.16-3.74) to 1.71 (95\% CI 0.92-3.18). A similar effect on the odds ratio was observed for current exposure to metoclopramide but not to proton pump inhibitors.

Conclusions Our algorithm to identify end-of-life care at home in the CPRD performed well, with only one false negative. The exclusion of misclassified cases of SCD reduced the magnitude of the odds ratios for SCD associated with domperidone and metoclopramide exposure by controlling protopathic bias.

\section{Key Points}

We developed an algorithm to identify end-of-life care in noninstitutionalized patients and excluded associated deaths from the analysis to address their misclassification as sudden cardiac death (SCD). The algorithm performed well, with only one false negative.

The exclusion of misclassified cases of SCD reduced the magnitude of the odds ratios for SCD associated with domperidone and metoclopramide exposure by controlling protopathic bias.

\section{Introduction}

The ascertainment of sudden cardiac death (SCD) in electronic health databases is challenging. A computer definition of SCD that was developed and validated using 
information collected in the Tennessee Medicaid program, with an overall positive predictive value (PPV) of $86.8 \%$, has been applied in several studies evaluating the risk of SCD associated with medications [1].

We used this computer definition to identify the cases in a recent retrospective study using data from the Clinical Practice Research Datalink (CPRD) in the UK that examined the relation of exposure to domperidone, a peripherally acting dopamine 2 receptor antagonist with both gastrokinetic and antiemetic actions, with the risk of SCD [2]. Other retrospective epidemiologic studies have examined this association and found an increased risk of serious ventricular arrhythmia and SCD in patients currently exposed to domperidone compared with patients not currently exposed [3-6]. However, this was the first such study to also include as a comparator metoclopramide, another medication with gastrokinetic and antiemetic actions but that has not been documented as associated with an increased risk of SCD. Inclusion of such a comparator was important because the indications for domperidone and metoclopramide were more similar than those for domperidone and the other comparator, proton pump inhibitors (PPIs).

Out-of-hospital SCD was ascertained by applying an adaptation of the algorithm published by Chung et al. [1], using information in CPRD GOLD data linked with Hospital Episode Statistics (HES) and death certificates collected by the Office for National Statistics (ONS). The study confirmed that, compared with non-use of any study drug, current domperidone use was associated with SCD and indicated that the increased risk was concentrated in the first 15 days of exposure in older individuals and in users of higher daily doses. An unexpected finding was a strong association between exposure to metoclopramide and an increased risk of SCD. When we pursued this association, we found that some of the SCD cases exposed to metoclopramide and some of those exposed to domperidone had been under palliative care shortly before death. Because SCD is, by definition, unexpected, these deaths were misclassified when the electronic algorithm identified them as SCD cases. We therefore reclassified all such SCD "cases" as non-cases and appropriately revised the estimates of association.

In this short communication, we evaluate the applicability of the validated computer definition of SCD [1] to electronic medical record data recorded from general practitioners in the UK that contribute data to the CPRD. This information may help future CPRD researchers improve the performance of the computer definition via appropriate identification of palliative care, including home-based end-of-life care in noninstitutionalized patients, that was not identified by the adapted computer case definition in our study. To address this misclassification, we created a separate algorithm to identify such cases and excluded them from the analysis.

\section{Methods}

The study population was derived from CPRD GOLD data and included individuals with permanent registration status in English practices whose data were linkable to HES and ONS data. Subjects entered the study cohort at their first exposure to a study drug (domperidone, any PPI medication, or metoclopramide) after at least 1 year of continuous enrollment in the CPRD GOLD database during the period 1 January 2005 through 31 December 2011. They had to be at least 2 years of age upon cohort entry. We excluded subjects with a diagnosis of cancer other than non-melanoma skin cancer and subjects residing in institutions. Follow-up of subjects continued until the earliest of (1) being transferred out of the practice, (2) last data collection date for each subject, (3) death, (4) receiving a code for diagnosis of cancer, (5) receiving a code for being transferred to an institutional setting, and (6) exiting the study.

All hospital episodes, each defined by admission date and discharge date plus 30 days after the discharge date, were identified. Person-time during these hospital episodes was excluded to ensure that time at risk occurred outside a hospital setting.

\subsection{Ascertainment of Sudden Cardiac Death (SCD)}

The study outcome was SCD, defined as an unexpected death from circulatory arrest, usually due to a cardiac arrhythmia, that occurred out of the hospital. The death had to be consistent with a cardiac cause, meaning absence of evidence of a noncardiac underlying process responsible for the death. Sudden cardiac death could present as (1) a witnessed sudden collapse with no pulse or respiration and death occurring within $1 \mathrm{~h}$ after the onset of cardiovascular symptoms or (2) an unwitnessed death in a person known to be alive and in stable condition in the $24 \mathrm{~h}$ before the death was reported $[1,3,7]$.

All deaths occurring during the observation period (2005-2011) were ascertained independently of exposure history by screening the electronic medical records for Read codes indicating death, by identifying patients with death recorded as the reason for transferring out of the practice, and by linkage of the CPRD GOLD with ONS death certificate information to obtain date and place of death, underlying cause of death, and all other causes of death listed on the death certificate [2]).

\subsubsection{Adaptation of the Validated Computer SCD Definition}

The original computer definition for SCD included the following components: (1) no evidence of a terminal institutional stay, (2) underlying cause of death consistent 
with SCD based on specific cause-of-death codes that are highly predictive of SCD, and (3) no terminal procedures (radiology, thrombolysis, general anesthesia) inconsistent with unresuscitated cardiac arrest [1]. In addition, we excluded patients with evidence of a life-threatening noncardiac illness from the cohort.

In our study, we excluded terminal institutional stays from the cohort. Therefore, we identified deaths with an underlying cause of death consistent with SCD based on the published list of International Classification of Diseases and Related Health Problems, 10th revision (ICD10) diagnosis codes [1]. From these, we retained only deaths that occurred within the study observation period with a date of death outside a hospital episode.

In the original computer SCD definition, deaths from cardiac arrests that occurred outside the hospital but were attended in an emergency department setting were included as cases of SCD unless terminal procedures in the emergency department were inconsistent with unresuscitated cardiac arrest [1]. In our study, it was not possible to apply this criterion and include such subjects because information on procedures that occurred during emergency department visits were available only in HES data, and HES data contained only those emergency visits that resulted in an inpatient admission.

In addition, we excluded cases of SCD that occurred within 30 days of an acute event: a medical encounter with acute stroke (ischemic or hemorrhagic), myocardial infarction, or heart failure as the principal, primary, or firstlisted diagnosis.

\subsubsection{Development of Algorithm for End-of-Life Home Care}

Using National Health Service (NHS) guidelines for palliative and end-of-life care [8], we identified expected cases of death in patients with evidence of palliative or terminal care shortly before the death by screening all suspected cases of SCD for the following codes:

- A palliative care code within 45 days before the date of death or

- Specific medication code(s) within 14 days before the date of death that included (1) a prescription for any of the following: any morphine except apomorphine, oxycodone injections, and fentanyl patches or other noninjectable fentanyl formulations and (2) a prescription for any of the following medications: cyclizine, haloperidol, levomepromazine, metoclopramide (intravenous formulation), midazolam, glycopyrronium, hyoscine hydrobromide, and hyoscine butylbromide.
The Read and product codes used are listed in the Electronic Supplementary Material.

\subsubsection{Review of Patient Profiles}

Detailed chronological electronic patient profiles were developed for all SCD cases that were reclassified by the end-of-life home care algorithm and were reviewed by two physicians (AA and CVL) to determine whether the algorithm excluded SCD cases that were truly expected deaths. Cases were reclassified as non-SCD cases if (1) codes were recorded for palliative care within 45 days before the date of death or (2) codes were recorded within 14 days before the date of death for at least two specific medications, one from each of the classes described previously, and were prescribed for end-of-life care.

Final cases of SCD were cardiac deaths with an underlying cause-of-death diagnosis code consistent with SCD and without an alternative noncardiac cause of death, such as pneumonia or substance overdose, that occurred in a noninstitutional setting. The case index date was the date of death as verified by the death certificate information.

\subsection{Analyses}

We describe the number and distribution of the cases according to the classification obtained with application of only the adapted validated computer case definition and with the addition of the algorithm for end-of-life home care. We present the multivariate adjusted effect of the study exposures to domperidone, PPIs, and metoclopramide on SCD using conditional logistic regression analysis for the two groups of cases identified. The mutually exclusive exposure categories to the study drugs were defined as follows:

- Current use: Time from date of prescription to end of calculated duration of exposure (duration of prescription plus 7 days).

- Past use: The 60 days after the end of a current use time window.

- Non-use: Study person-time outside of current use or past use windows.

\section{Results}

The final study cohort comprised 681,104 subjects. Among these, 58,647 deaths were identified in the study cohort from the ONS linkage. Details on cohort creation are provided in the publication of the main study [2]. 


\subsection{Electronically Identified SCD Cases in the Overall Cohort}

Using the adaptation of the algorithm developed and validated by Chung et al. [1], 9765 of all identified deaths had a cardiovascular diagnosis as the underlying cause, and 3509 of these occurred outside a hospital episode during the study follow-up. Because 65 of these had an alternative explanation as a cause of death, a total of 3444 were initially classified as out-of-hospital SCD.

\subsubsection{Application of the Algorithm for End-of-Life Home Care to All Identified SCD Cases}

In a further step, 163 deaths were identified as expected deaths by our algorithm developed to identify palliative/ end-of-life home care. After review of the patient profiles, 162 were classified as expected deaths because of evidence that the patient received palliative care or end-of-life treatment. One, a case of low back pain treated with morphine, was a false negative $(1$ of $163=0.6 \%$; $95 \%$ confidence interval [CI] 0.03-2.98; exact confidence limits calculated with Episheet) [9].

Finally, 3282 cases met the operational study definition of out-of-hospital SCD. Reclassification of cases based on the application of the algorithm is shown in Table 1.

\subsection{Effect of the Misclassification on Study Relative Risk Estimates}

Table 2 shows the study odds ratios estimated for current exposure to domperidone, metoclopramide, and PPIs before and after reclassification of cases. Of the initial 3444 SCD cases identified by applying the adapted version of the computer definition by Chung et al. [1], 47 could not be matched with at least one control, so the total number of
Table 1 Attrition table for deaths

\begin{tabular}{lr}
\hline Category of death & $\begin{array}{c}\text { Number of } \\
\text { patients }\end{array}$ \\
\hline Total deaths in study cohort from ONS linkage & 58,647 \\
Deaths with selected cardiovascular diagnosis as underlying cause (diagnosis code from & 9765 \\
Chung et al. [1]) & 5664 \\
Deaths occurring outside a hospital episode & 3509 \\
Deaths during study period/patient follow-up & 65 \\
Deaths that have an alternative noncardiac cause of death & 3444 \\
Total number of cases of SCD, by adapted computer definition of Chung et al. [1] & 162 \\
Palliative/terminal care within 45 days prior to date of death & 3282 \\
\hline Total number of final cases of SCD & \\
\hline
\end{tabular}

ONS Office for National Statistics, SCD sudden cardiac death

Table 2 Risk of sudden cardiac death, according to the two sets of cases and controls, by study exposures, nested case-control analysis results of multivariable conditional logistic regression

\begin{tabular}{|c|c|c|c|c|}
\hline \multirow[t]{2}{*}{ Exposure category (categories are mutually exclusive) } & \multicolumn{2}{|c|}{ SCD cases-SET $1^{\text {a }}(N=3397)$} & \multicolumn{2}{|c|}{ SCD cases-SET $2^{\mathrm{b}}(N=3239)$} \\
\hline & $N(\%)$ & Adjusted $\mathrm{OR}^{\mathrm{c}}(95 \% \mathrm{CI})$ & $N(\%)$ & Adjusted $\mathrm{OR}^{\mathrm{c}}(95 \% \mathrm{CI})$ \\
\hline Current exposure to domperidone & $31(0.9)$ & $2.09(1.16-3.74)$ & $28(0.9)$ & $1.71(0.92-3.18)$ \\
\hline Current exposure to PPI & $2007(59.1)$ & $1.32(1.18-1.48)$ & $1935(59.7)$ & $1.35(1.21-1.51)$ \\
\hline Current exposure to metoclopramide & $48(1.4)$ & $4.93(2.82-8.64)$ & $37(1.1)$ & $4.31(2.33-7.98)$ \\
\hline Current combined exposure ${ }^{\mathrm{d}}$ & $120(3.5)$ & $3.08(2.20-4.31)$ & $96(3.0)$ & $2.68(1.87-3.83)$ \\
\hline Past exposure to any study drug & $367(10.8)$ & $1.23(1.04-1.46)$ & $341(10.5)$ & $1.20(1.01-1.43)$ \\
\hline No exposure to any study drug & $824(24.3)$ & Reference & $802(24.8)$ & Reference \\
\hline
\end{tabular}

$C I$ confidence interval, $O R$ odds ratio, $P P I$ proton pump inhibitors, SCD sudden cardiac death

${ }^{\text {a }}$ SET 1 = cases of SCD, using adapted Chung et al. [1] algorithm

b SET 2 = cases of SCD, using both the adapted Chung et al. [1] algorithm and the study palliative/terminal home care algorithm

c OR matched for age, sex, and practice, and adjusted for covariates: history of serious ventricular arrhythmia, myocardial infarction, heart failure, valvular heart disease including valve replacement, cardiomyopathy, other arrhythmia or conduction disorder, epilepsy, depression, group 2 QTc-prolonging drugs, drugs that affect hERG, digoxin, diuretics, laxatives, beta-blockers, body mass index, alcohol use, smoking history, number of general practitioner visits, and number of hospital admissions

${ }^{\mathrm{d}}$ Current exposure to more than one study drug: domperidone + PPI, domperidone + metoclopramide, PPI + metoclopramide, or domperidone + PPI + metoclopramide 
cases in the nested case-control analysis was 3397. Our final classification via the additional application of the algorithm for palliative/end-of-life home care resulted in 3282 cases of SCD, 43 of which could not be matched with at least one control, so the total number of cases in the nested case-control analysis was 3239 .

The exclusion of cases with palliative or end-of-life home care did not modify dramatically the distribution of cases among each exposure category. The odds ratio for current PPI use compared with no use of any study drug was similar for each classification process: $1.32(95 \% \mathrm{CI}$ 1.18-1.48) before and 1.35 (95\% CI 1.21-1.51) after excluding cases with end-of-life home care.

The exclusion of such cases did appreciably change the estimate for current exposure to domperidone, which was the estimate of primary interest in the study. Compared with non-use of study medications, the original estimate was 2.09 (95\% CI 1.16-3.74), and the corrected estimate was 1.71 (95\% CI 0.92-3.18). A similar effect was observed for current exposure to metoclopramide compared with non-use of study medications; the estimate changed from 4.93 (95\% CI 2.82-8.64) to 4.31 (95\% CI 2.33-7.98) after the exclusion of the misclassified cases.

These results were expected because domperidone and metoclopramide can be used for palliative care in diseases other than cancer. Nausea and vomiting are among the commonest symptoms in patients under palliative care, and UK guidelines indicate that side effects of opioid analgesics used for palliative care are nausea and vomiting, which are common in opioid-naïve patients and can be prevented by access to an antiemetic, e.g., metoclopramide [8].

\section{Conclusion}

We have evaluated the applicability of a published validated computer definition of SCD, developed using Medicaid data in the USA, to a different data source (CPRD) and healthcare system (UK). The use of detailed information recorded by general practitioners from the UK helped us to improve the applicability of the computer algorithm by additional identification of expected deaths due to home-based palliative end-of-life care. Our algorithm to identify home-based palliative care performed well, with only one false negative. The identification and exclusion of these misclassified cases of SCD reduced the magnitude of the odds ratios for SCD associated with domperidone and metoclopramide exposure by controlling protopathic bias. This bias may be present when estimating the risk of SCD associated with any medications used for palliative or endof-life care. We would recommend using all available relevant information to identify and exclude subjects who receive palliative end-of-life care from database studies that examine the association of exposure to a medication with the risk of SCD.

Acknowledgments The authors thank Amy Ladner for expert project management, Susana Perez-Gutthann for review, and Adele Monroe for expert editorial support.

\section{Compliance with Ethical Standards}

Disclosure of potential conflict of interest All authors have completed the conflicts of interest disclosure form. All authors received financial support from Johnson \& Johnson for the submitted work. DF is a full-time employee of Johnson \& Johnson, holds stock and stock options in the company, and holds pension rights from Johnson \& Johnson. CVL, AA, CBJ, LJM, and KJR have had no financial relationships with organizations that might have an interest in the submitted work in the previous 3 years; none of the authors have other relationships or activities that could appear to have influenced the submitted work.

Research involving human participants and/or animals Ethical approval: All procedures performed in studies involving human participants were in accordance with the ethical standards of the institutional and/or national research committee and with the 1964 Helsinki declaration and its later amendments or comparable ethical standards. For this type of study, formal consent is not required. The study was determined exempt from full review by the institutional review board of RTI International. The study protocol received approval for database research from the Independent Scientific Advisory Committee of the Medicines and Healthcare Products Regulatory Agency (UK).

Open Access This article is distributed under the terms of the Creative Commons Attribution-NonCommercial 4.0 International License (http://creativecommons.org/licenses/by-nc/4.0/), which permits any noncommercial use, distribution, and reproduction in any medium, provided you give appropriate credit to the original author(s) and the source, provide a link to the Creative Commons license, and indicate if changes were made.

\section{References}

1. Chung CP, Murray KT, Stein CM, Hall K, Ray WA. A computer case definition for sudden cardiac death. Pharmacoepidemiol Drug Saf. 2010;19(6):563-72.

2. Arana A, Johannes CB, McQuay LJ, Varas-Lorenzo C, Fife D, Rothman KJ. Risk of out-of-hospital sudden cardiac death in users of domperidone, proton pump inhibitors, or metoclopramide: a population-based nested case-control study. Drug Saf. 2015;38(12):1187-99.

3. Straus SM, Sturkenboom MC, Bleumink GS, Dieleman JP, van der Lei J, de Graeff PA, et al. Non-cardiac QTc-prolonging drugs and the risk of sudden cardiac death. Eur Heart J. 2005;26(19):2007-12.

4. van Noord C, Dieleman JP, van Herpen G, Verhamme K, Sturkenboom MC. Domperidone and ventricular arrhythmia or sudden cardiac death: a population-based case-control study in the Netherlands. Drug Saf. 2010;33(11):1003-14.

5. Johannes CB, Varas-Lorenzo C, McQuay LJ, Midkiff KD, Fife D. Risk of serious ventricular arrhythmia and sudden cardiac death in a cohort of users of domperidone: a nested case-control study. Pharmacoepidemiol Drug Saf. 2010;19(9):881-8. 
6. Chen HL, Hsiao FY. Domperidone, cytochrome P450 3A4 isoenzyme inhibitors and ventricular arrhythmia: a nationwide case-crossover study. Pharmacoepidemiol Drug Saf. 2015;24(8):841-8.

7. Ray WA, Chung CP, Murray KT, Hall K, Stein CM. Atypical antipsychotic drugs and the risk of sudden cardiac death. N Engl J Med. 2009;360(3):225-35.
8. North of England Cancer Network. Palliative and end of life care guidelines for cancer and non-cancer patients, 3rd ed. 2012. http:// www.nesra.co.uk/files/acutepain/NECNPalliativeCareGuidelines Booklet2012.pdf. Accessed 14 Aug 2015.

9. Rothman KJ. Episheet-spreadsheets for the analysis of epidemiologic data. 4 Oct 2012. http://www.krothman.org/episheet.xls. Accessed 14 Aug 2015. 